\title{
Epidemiological typing of coagulase-negative staphylococci by pyrolysis mass spectrometry
}

\author{
R. FREEMAN, M. GOODFELLOW, A. C. WARD, S. J. HUDSON*, \\ F. K. GOULDt and N. F. LIGHTFOOT‡
}

Department of Microbiology, University of Newcastle upon Tyne, Queen Elizabeth Hospital, Gateshead, tFreeman Hospital, Newcastle upon Tyne, and $\ddagger$ Regional Public Health Laboratory, Newcastle upon Tyne

\begin{abstract}
Summary. Pyrolysis mass spectrometry (PMS) was evaluated for the epidemiological typing of coagulase-negative staphylococci (CNS) in situations in which it was necessary to distinguish between repeated isolation of the same strain from a single patient (genuine infection) and coincidental isolation of unrelated strains (contamination). Thirteen CNS isolates were examined, consisting of five pairs, each pair isolated from a single patient, and three unrelated strains. PMS analysis gave results equivalent to a conventional typing system comprising antibiogram, biotype and plasmid profile analysis. Both methods facilitate differentiation between genuine infection with CNS and the isolation of contaminants. The speed, reproducibility, versatility and relatively low cost of PMS suggest that it may be a valuable new technique for the epidemiological typing of CNS in routine clinical settings.
\end{abstract}

\section{Introduction}

Pyrolysis mass spectrometry (PMS) has been reported to be a rapid, sensitive and reproducible method for interstrain comparisons of micro-organisms. ${ }^{1,2}$ The technique has recently been applied to clinical isolates of Streptococcus pyogenes $^{3,4}$ and Salmonella ${ }^{4}$ and was shown to distinguish isolates of an outbreak or epidemic strain from sporadic isolates of the same species.

Another potential use of PMS is in comparing several isolates from an individual patient. A common problem associated with the isolation of organisms such as coagulase-negative staphylococci (CNS) is in assessing their clinical significance. The ability to show that different isolates of CNS, obtained from more than one site in an individual patient or from the same site (especially blood cultures) on successive occasions, are either indistinguishable or dissimilar would be a considerable help in distinguishing between contamination of such samples with skin organisms and the possibility of a genuine infection. Plasmid profiling has been used in these circumstances but is not widely available. ${ }^{10}$ This study was designed to test the usefulness of PMS for this purpose.

\section{Materials and methods}

\section{Bacterial strains and media}

Thirteen isolates of CNS were collected over a period of several weeks from the diagnostic micro-

Received 12 June 1990; accepted 1 Aug. 1990. biology laboratory at Freeman Hospital, Newcastle upon Tyne. Ten of these isolates comprised five pairs of organisms in which there was presumptive evidence that the two isolates might be identical. In four cases (patients A-D) with intravenous catheters in situ, one isolate was from a culture of peripheral blood, taken from an uncannulated vein, and the second was from the tip of the catheter after its removal. In the fifth instance (patient $E$ ) the paired isolates were from blood cultures taken on successive days from a patient with suspected prosthetic valve endocarditis. The diagnosis of endocarditis was subsequently confirmed at operation, although culture of the excised valve was negative, probably due to appropriate antibiotic therapy before surgery. The other three isolates of CNS were from peritoneal dialysis effluents in distinct episodes of peritonitis in three different patients $(F$, $\mathrm{G}, \mathrm{H})$ undergoing continuous ambulatory peritoneal dialysis and were, therefore, epidemiologically unrelated to each other or to any of the paired isolates.

CNS strains were stored in glycerol broth at $-20^{\circ} \mathrm{C}$. To minimise variability, all subsequent separate studies (antibiogram, biotype, plasmid profiles and PMS) were performed on single subcultures taken directly from this primary source rather than on serial subcultures.

\section{Antibiogram determination}

After subculture on to Nutrient Agar (Lab M, Bury, Lancs) and checking for purity, isolates were tested by the comparative disk-diffusion method ${ }^{5}$ for sensitivity to the following antibiotics: penicillin ( $p, 5 \mathrm{IU}$ ), methicillin $(\mathrm{m}, 10 \mu \mathrm{g})$, gentamicin $(\mathrm{g}, 10 \mu \mathrm{g})$, amikacin 
(a, $30 \mu \mathrm{g}$ ), streptomycin (s, $25 \mu \mathrm{g})$, neomycin (n, $30 \mu \mathrm{g}$ ), vancomycin $(\mathrm{v}, 30 \mu \mathrm{g})$, tetracycline $(\mathrm{t}, 50 \mu \mathrm{g})$, erythromycin (e, $5 \mu \mathrm{g}$ ), clindamycin (c, $2 \mu \mathrm{g})$, fusidic acid (f, $10 \mu \mathrm{g})$, rifampicin ( $2 \mu \mathrm{g})$, trimethoprim (tr, $2 \cdot 5 \mu \mathrm{g}$ ), chloramphenicol (ch, $50 \mu \mathrm{g})$, novobiocin $(5 \mu \mathrm{g})$, bacitracin (b, 0.04 units) and furazolidone $(100 \mu \mathrm{g})$. Sensitivity tests were read after incubation for $24 \mathrm{~h}$ at $37^{\circ} \mathrm{C}$ except for tests of methicillin sensitivity which were incubated at $30^{\circ} \mathrm{C}$. Isolates were recorded as either sensitive or resistant; intermediate zone sizes were recorded as resistant. For bacitracin testing, any zone size, however small, was regarded as indicating sensitivity.

\section{Biotyping}

All isolates were examined by the API-Staph method (API Products Ltd, Basingstoke, Hants) in a single batch of tests, omitting the lysostaphin test. The strips were inoculated and read after incubation for $24 \mathrm{~h}$ at $37^{\circ} \mathrm{C}$, according to the manufacturers' instructions.

\section{Plasmid profiling}

Plasmid profile analysis was performed on the 10 organisms comprising the five clinically related pairs. Plasmid DNA was extracted following lysis of the cells by lysostaphin and lysozyme (Sigma) ${ }^{6}$ and analysed by electrophoresis. ${ }^{7}$ Each distinguishable pattern was assigned an arbitrary number (P1, P2, etc.) to allow comparisons between the two members of each pair and between pairs.

\section{Pyrolysis mass spectrometry (PMS)}

All 13 isolates were subcultured on to single plates from one batch of nutrient agar which were incubated overnight at $37^{\circ} \mathrm{C}$ in identical conditions. Isolates were identified by laboratory number and then coded as CNS 1-13 by one of the investigators. PMS analysis was performed by the other investigators who were unaware of the identity of the individual isolates or of the results of antibiogram, biotype and plasmid profile results until after completion of the PMS analysis.

A well-separated colony of the isolate to be tested was sampled with a flamed straight wire. Care was taken to avoid contamination with the medium. The material was smeared on to a pyrolysis foil (Horizon Instruments Ltd, Heathfield, Kent) to give a uniform surface coating and the foil was inserted into a pyrolysis tube (Horizon Instruments). Assembled tubes were heated at $80^{\circ} \mathrm{C}$ for $5 \mathrm{~min}$ in a hot air oven. Each sample was prepared in triplicate.

All 39 samples were processed in a single batch on a Horizon Instruments PYMS 200X pyrolysis mass spectrometer. The time required for the analysis of each sample was c. 2 min and pyrolysis was at a Curie point of $530^{\circ} \mathrm{C}$ for a period of $4 \mathrm{~s}$. Products were ionised by collision with a crossing beam of low energy
$(25 \mathrm{eV})$ electrons, and the ions were separated in a quadrupole mass spectrometer which scanned the pyrolysate at intervals of $0.35 \mathrm{~s}$ from the start of pyrolysis. Integrated ion counts at unit mass intervals from 11 to 200 were recorded on floppy disk, together with the pyrolysis sequence number and total ion count for each individual sample.

\section{Data analysis}

Spectra were normalised by an iterative technique to eliminate variation due to differences in the amount of sample pyrolysed. After normalisation, replicate spectra of the same strain were labelled as distinct groups and analysed for between-strain variation to select the mass ion peaks showing the greatest ratio of between-strain to within-strain variation. The 30 mass ion peaks showing the greatest discrimination between strains were then subjected to principal component analysis and canonical variate analysis, resulting in a table of Mahalanobis distances. These data were finally used in an UPGMA analysis to produce a dendrogram. ${ }^{8}$ The apparatus, technique and principles involved have been described previously. ${ }^{9}$

\section{Results}

The table contains details of the isolates studied, the presumptive clinical associations between pairs of isolates and the results of antibiogram and biotype testing for all the isolates, and the results of plasmid profiling for those isolates comprising the five clinically matched pairs. The figure shows a dendrogram based on average linkages obtained by processing the Mahalanobis distances data by UPGMA analysis. Isolates 1, 2 and 5 were identified as a group separate from the remainder of the strains, although isolates 1 and 2 appeared to be more similar to each other than either was to isolate 5. All three isolates were Staphylococcus haemolyticus, but only isolates 1 and 2 had the same antibiogram. These two isolates, which also gave identical plasmid-profile patterns, were isolated from the catheter tip and blood culture of the same patient; isolate 5, however, was isolated from a completely unrelated patient. Within the remainder of the organisms (all of which were identified as $S$. epidermidis by API-Staph), the PMS-derived dendrogram indicates that isolates 4 and 7 were more closely similar to each other than to any other isolates, as were isolates 8 and 9 . Isolates 4 and 7 were from blood cultures taken on successive days from a patient with prosthetic valve endocarditis and had identical antibiograms, biotypes and plasmid profiles; isolates 8 and 9 were from the catheter tip and accompanying blood culture taken from one patient and again had identical antibiograms, biotypes and plasmid profiles.

In the two remaining instances in which pairs of CNS isolates were thought to be clinically related (isolates 10 and 11 ; isolates 6 and 12), the biotypes 
Table. Clinical sources, antibiograms, biotypes and plasmid profiles of 13 isolates of CNS

\begin{tabular}{|c|c|c|c|c|}
\hline Patient/source & $\begin{array}{l}\text { Isolate } \\
\text { code }\end{array}$ & Antibiogram* & Species/biotype $†$ & $\begin{array}{c}\text { Plasmid* } \\
\text { profile }\end{array}$ \\
\hline A/blood & 1 & b pmgasnttmch & S. haemolyticus 6632051 & P1 \\
\hline A/catheter tip & 2 & b pmgasnttm ch & S. haemolyticus 6632051 & P1 \\
\hline B/blood & 6 & b pm g a n tm ch & S. epidermidis 6606013 & P3 \\
\hline $\mathrm{B} /$ catheter tip & 12 & b mg a s nttm & S. epidermidis 6606013 & P7 \\
\hline C/blood & 8 & b pe & S. epidermidis 6606113 & P4 \\
\hline C/catheter tip & 9 & b pe & S. epidermidis 6606113 & P4 \\
\hline D/blood & 11 & b p m g a n e cl ch & S. epidermidis 6606113 & P6 \\
\hline $\mathrm{D} /$ catheter tip & 10 & b pmgantecl tm & S. epidermidis 6606113 & P5 \\
\hline E/blood & 4 & $\mathrm{~b}$ & S. epidermidis 6606113 & $\mathbf{P} 2$ \\
\hline E/blood & 7 & $\mathrm{~b}$ & S. epidermidis 6606113 & P2 \\
\hline $\mathrm{F} /$ peritoneal dialysis effluent & 3 & $\mathrm{~b} p \mathrm{~m}$ & S. epidermidis 6606113 & ND \\
\hline $\mathrm{G} /$ peritoneal dialysis effluent & 5 & b m g s n tm ch & S. haemolyticus 6632051 & ND \\
\hline $\mathrm{H} /$ peritoneal dialysis effluent & 13 & bpmgant tm & S. epidermidis 6606113 & ND \\
\hline
\end{tabular}

* See Materials and methods for abbreviations.

+ API-Staph profiles.

ND, not done.

were identical but antibiograms suggested that the organisms were not appropriately paired and this was supported by differences in plasmid profile patterns within each pair. The PMS analysis (figure) confirmed these dissimilarities. Isolate 10 was more similar to the sporadic isolate 13 than to isolate 11 which in turn was more similar to isolate 12 than to isolate 10 . In the second case, isolate 6 was more closely similar to isolate 3 , a sporadic isolate, than to isolate 12 with which it was clinically associated.

\section{Discussion}

Assessing the similarity or otherwise of a series of isolates of CNS from a single patient is a common problem in clinical microbiology. There is a need for a highly discriminatory and reliable typing system for CNS which should preferably also be inexpensive and simple enough to be performed in most laboratories. A recent attempt to establish a practical typing system ${ }^{10}$ stressed the value of the antibiogram, which together with estimation of biotype by the API-Staph method, achieved $87 \%$ of the discriminatory power of the assessed system. Taking discrimination to levels greater than $90 \%$, however, was found to require either plasmid profiling or phage typing, both of which are technically demanding and beyond the capabilities of a routine hospital laboratory.

In the present study, antibiograms, biotypes and plasmid profiles of five clinically-related pairs of CNS, showed that in two instances the organisms within each pair were different, whereas in the remaining three cases the organisms within each pair were

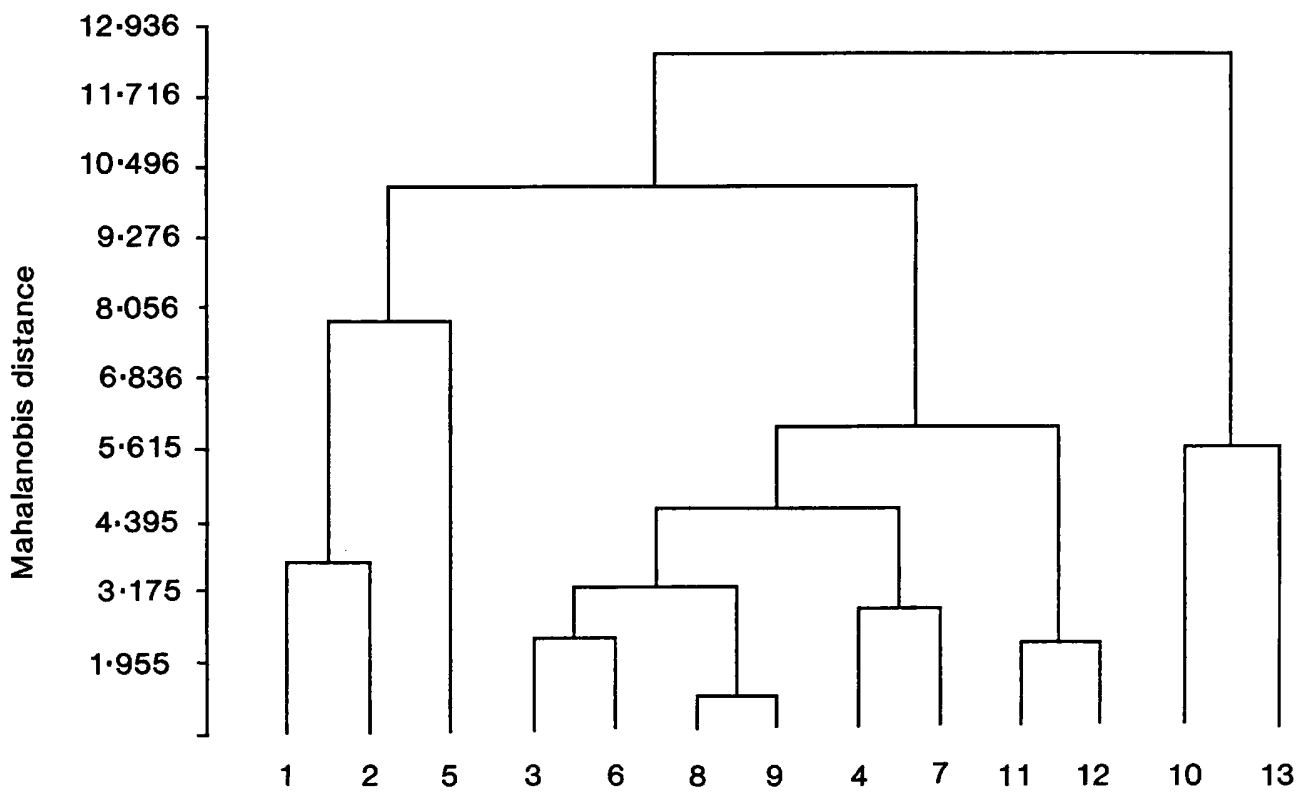

Strain no.

Figure. Dendrogram based on PyMS data. The dissimilarity measure is Mahalanobis distance. The clustering method was UPGMA. 
indistinguishable. PMS examination of the same organisms produced inter-strain comparisons which were a mathematical expression of compositional differences between the organisms in each pair. These results formed the basis of a limited taxonomic study of the 10 strains, having the advantage of using unweighted characteristics in the form of the mass ion measurements. Because the PMS approach relies on comparing mass spectra of the strains under investigation for relative similarity it was felt important to include three epidemiologically unrelated isolates of CNS as reference points in each batch against which similarity and difference could be measured. In the two instances in which the conventional systems showed that the CNS isolates had been inappropriately paired, PMS analysis demonstrated that the isolates were more similar to unrelated isolates than to each other. For the remaining three pairs, which were indistinguishable by the conventional typing system, PMS analysis showed much higher levels of similarity between the organisms within each pair than with other unrelated isolates. As a laboratory tool for assisting in the interpretation of blood-culture results

\section{References}

1. Gutteridge CS, Norris JR. The application of pyrolysis techniques to the identification of micro-organisms. $J$ Appl Bacteriol 1979; 47: 5-43.

2. Shute LA, Berkeley RCW, Norris JR, Gutteridge CS. Pyrolysis mass-spectrometry in bacterial systems. In: Goodfellow M, Minnikin DE (eds) Chemical methods in bacterial systematics. (The Society for Applied Bacteriology Technical Series No. 20). London, Academic Press. 1985: 95114.

3. Magee JT, Hindmarch JM, Burnett IA, Pease A. Epidemiological typing of Streptococcus pyogenes by pyrolysis mass spectrometry. J Med Microbiol 1989; 30: 273-278.

4. Freeman R, Goodfellow M, Gould FK, Hudson SJ, Lightfoot NF. Pyrolysis-mass spectrometry (Py-MS) for the rapid epidemiological typing of clinically significant bacterial pathogens. J Med Microbiol 1990; 32: 283-286.

5. Brown D, Blowers R. Disc methods of sensitivity testing and other semiquantitative methods. In: Reeves DS, Phillips I, Williams JD, Wise R (eds) Laboratory methods in
PMS results were comparable to the findings of more conventional typing methods. Direct application of PMS may be an alternative to antibiograms and plasmid profiles or phage typing.

PMS is a rapid, reproducible, and relatively inexpensive technique. After the initial capital investment (c. $£ 80000$ ) the technique requires few consumables and little labour. The cost of analysing individual samples has been estimated to be less than $£ 1 .{ }^{11}$ Furthermore, PMS apparatus need not be dedicated to any one group of micro-organisms and the ability to sequentially examine different species by a single method is attractive. The technique has been used successfully to investigate isolates associated with outbreaks. $^{3,4}$ The results of this small study suggest that PMS can also be used to provide clinically-useful information when attempting to interpret the repeated isolation of the same bacterial species, particularly one often found as a contaminant, from a single patient.

We are grateful to Dr C. R. Harwood and Mrs D. Collier of the Microbiology Department, University of Newcastle upon Tyne, for the results of plasmid profile analyses.

antimicrobial chemotherapy. Edinburgh, Churchill Livingstone. 1978: 30

6. Birnboim HC, Doly J. A rapid alkaline extraction procedure for screening recombinant plasmid DNA. Nucleic Acids Res 1979; 7: 1513-1523.

7. Meyers JA, Sanchez D, Elwell LP, Falkow S. Simple agarose gel electrophoretic method for the identification and characterization of plasmid deoxyribonucleic acid. $J$ Bacteriol 1976; 127: 1529-1537.

8. Sokal RR, Michener CD. A statistical method for evaluating systematic relationships. University of Kansas Science Bulletin 1958; 38: 1409-1438.

9. Aries RE, Gutteridge CS, Ottley TW. Evaluation of a low-cost, automated pyrolysis-mass spectrometer. J Anal Appl Pyrolysis 1986; 9: 81-98.

10. Ludlam HA, Noble WC, Marples RR, Phillips I. The evaluation of a typing scheme for coagulase-negative staphylococci suitable for epidemiological studies. J Med Microbiol 1989; 30: $161-165$.

11. Magee JT, Hindmarch JM, Bennett KW, Duerden BI, Aries RE. A pyrolysis mass spectrometry study of fusobacteria. J Med Microbiol 1989; 28: 227-236. 